\title{
Application of pattern search method to power system valve-point economic load dispatch
}

\author{
J.S. Al-Sumait ${ }^{\text {a }}$, A.K. AL-Othman ${ }^{\text {b,* }}$, J.K. Sykulski ${ }^{\text {a }}$ \\ ${ }^{a}$ Electronics and Computer Science School, University of Southampton, Highfield Southampton SO17 1BJ, UK \\ ${ }^{\mathrm{b}}$ Electrical Engineering Department, College of Technological Studies, Alrawda 73452, P.O. Box 33198, Kuwait
}

Received 13 September 2006; received in revised form 8 June 2007; accepted 9 June 2007

\begin{abstract}
Direct search (DS) methods are evolutionary algorithms used to solve constrained optimization problems. DS methods do not require any information about the gradient of the objective function at hand, while searching for an optimum solution. One of such methods is pattern search (PS) algorithm. This study presents a new approach based on a constrained pattern search algorithm to solve well-known power system economic load dispatch problem (ELD) with valve-point effect. For illustrative purposes, the proposed PS technique has been applied to various test systems to validate its effectiveness. Furthermore, convergence characteristics and robustness of the proposed method has been assessed and investigated through comparison with results reported in literature. The outcome is very encouraging and proves that pattern search (PS) is very applicable for solving power system economic load dispatch problem.
\end{abstract}

(c) 2007 Elsevier Ltd. All rights reserved.

Keywords: Economic-load dispatch; Valve-point effect; Direct search method; Pattern search method; Evolutionary algorithms (EA); Optimization

\section{Introduction}

Scarcity of energy resources, increasing power generation cost and ever-growing demand for electric energy necessitates optimal economic dispatch in today's power systems. The main objective of economic dispatch is to reduce the total power generation cost, while satisfying various equality and inequality constraints. Traditionally in ED problems, the cost function for generating units has been approximated as a quadratic function.

A wide variety of optimization techniques have been applied to solving economic load dispatch problems (ELD). Some of these techniques are based on classical optimization methods, while others use artificial intelligence methods or heuristic algorithms. Many references present the application of classical optimization methods,

\footnotetext{
${ }^{*}$ Corresponding author. Tel.: +965 2314323; fax: +965 4816568.

E-mail addresses: jas2@soton.ac.uk (J.S. Al-Sumait), ak.alothman@ paaet.edu.kw (A.K. AL-Othman), ks@soton.ac.uk (J.K. Sykulski).
}

such as linear programming or quadratic programming, to solve ELD problems [1,2]. Such classical optimization methods are highly sensitive to staring points and often converge to local optimum or diverge altogether. Linear programming methods are fast and reliable, but have a disadvantage associated with the piecewise linear cost approximation. Non-linear programming methods have known problems of convergence and algorithmic complexity. Newton based algorithms have difficulty with handling a large number of inequality constraints [3]. Methods based on artificial intelligence techniques, such as artificial neural networks, have also been applied successfully and are reported for example in [4,5]. Lately, many heuristic search techniques, such as particle swarm optimization [3] and genetic algorithms [6,7], have been considered in the context of the ELD problems. Finally, hybrid methods have been developed [8], where the conventional Lagrangian relaxation approach, first order gradient method and multi-pass dynamic programming are combined together. In addition, authors in [9] have presented an alternative 
form of hybrid method to solve a valve-point ELD problem. In [9] deferential evolution (DE) was the primary optimizer exploiting its global search capabilities, sequential quadratic programming (SQP) was then employed to fine tune the best solution provided by DE.

Recently, a particular family of global optimization methods, introduced and developed by researchers in 1960 [10], has received a great attention, namely the direct search methods. Direct search methods are simply structured to explore a set of points, around the current position, looking for a point that has smaller objective value than the current one. This family includes pattern search (PS) algorithms, simplex methods (SM) (different from the simplex used in linear programming), powell optimization (PO) and others [11].

Direct search methods, as apposed to more standard optimization methods, are often called derivative-free optimization methods, as they do not require any information about the gradient or higher derivatives of the objective function to search for an optimal solution. Therefore direct search methods may very well be used to solve non-continues, non-differentiable and multimodal, i.e. multiple local optima, optimization problems. Since the economic dispatch is one such problem, then the proposed method appears to be a good choice.

The main objective of this study is to introduce the use of pattern search (PS) optimization technique to the subject of power system economic load dispatch. In this paper, the PS method has been employed to solve economic dispatch problem with a valve-point effect. A valve-point effect is the rippling effect added to the generating unit curve when each steam admission valve in a turbine starts to open. Moreover, to assure accurate results for this model, an additional term representing the valve-point effect should be added to the cost function [12]. The addition of the valve-point effect poses a more challenging task to the proposed method since it increases the non-linearity of the search space as well as the number of local minima. (see Fig. 1).

The paper is organized as follows: Section 2 introduces the problem formulation. Section 3 presents a description

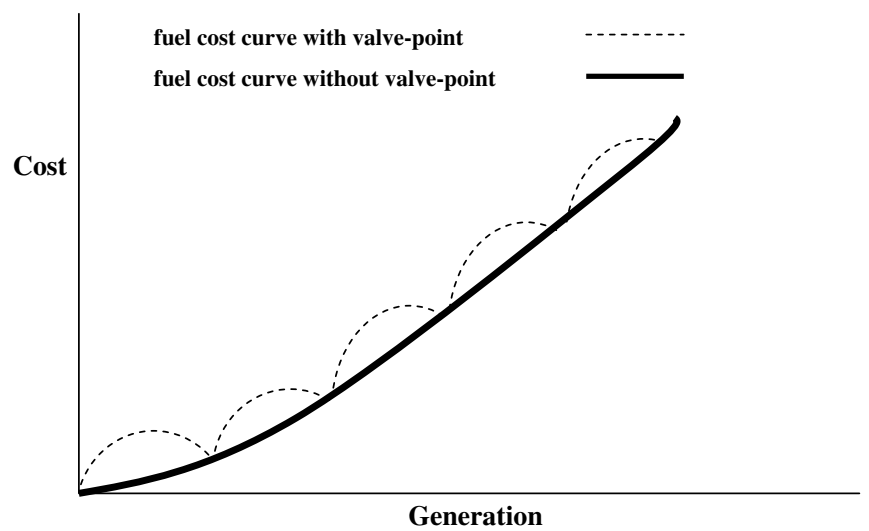

Fig. 1. The valve-point effect. of the proposed PS algorithm. Analysis and test results are presented in Section 4, followed by concluding remarks.

\section{Problem formulation}

The traditional formulation of the economic load dispatch problem is a minimization of summation of the fuel costs of the individual dispatchable generators subject to the real power balanced with the total load demand as well as the limits on generators outputs. In mathematical form the problem can be stated as:

$F=\sum_{i=1}^{N} F_{i}\left(P_{i}\right)$

The incremental fuel cost function of the generation units with valve-point loading is represented as follows [6]:

$F_{i}\left(P_{i}\right)=a_{i} P_{i}^{2}+b_{i} P_{i}+c_{i}+\left|e_{i} \times \sin \left(f_{i} \times\left(P_{\mathrm{g} i(\min )}-P_{i}\right)\right)\right|$

Subject to

$\sum_{i=1}^{N} P_{\mathrm{g} i}=P_{\mathrm{D}}+P_{\mathrm{L}}$

$P_{\mathrm{g} i_{(\min )}}<P_{\mathrm{g} i}<P_{\mathrm{g} i_{(\max )}}, i \in N_{\mathrm{s}}$

where $F$ is the system overall cost function; $N$ is the number of generators in the system; $d_{i}, b_{i}, c_{i}$ the constants of fuel function of generator number $i ; e_{i}, f_{i}$ the constants of the valve-point effect of generator number $i ; P_{\mathrm{g} i}$ the active power generation of generator number $i ; P_{\mathrm{D}}$ the total power system demand; $P_{\mathrm{L}}$ the total system transmission losses; $P_{\mathrm{g} i_{(\min )}}$ the minimum limit on active power generation of generator $i ; P_{\mathrm{g}_{i \max }}$ the maximum limit on active power generation of generator $i ; N_{\mathrm{s}}$ the set of generators in the system.

The sinusoidal term added to the fuel cost function which models the valve-point effect introduces ripples to heat-rate curve and therefore introducing more local minima to the search space.

It should be noted that the system losses are ignored for all test systems considered in this study for simplification purposes.

\section{Pattern search method}

The pattern search (PS) optimization routine is an evolutionary technique that is suitable to solve a variety of optimization problems that lie outside the scope of the standard optimization methods. Generally, PS has the advantage of being very simple in concept, easy to implement and computationally efficient. Unlike other heuristic algorithms, such as genetic algorithms [13,14], PS possesses a flexible and well-balanced operator to enhance and adapt the global and fine tune local search. A useful review of direct search methods for unconstrained optimization is presented in [11], where the authors give a modern perspec- 
tive on the classical family of derivative-free algorithms, focusing on the development of direct search methods.

The pattern search (PS), algorithm proceeds by computing a sequence of points that may or may not approaches to the optimal point. The algorithm starts by establishing a set of points called mesh, around the given point. This current point could be the initial starting point supplied by the user or it could be computed from the previous step of the algorithm. The mesh is formed by adding the current point to a scalar multiple of a set of vectors called a pattern. If a point in the mesh is found to improve the objective function at the current point, the new point becomes the current point at the next iteration.

This maybe better explained by the following:

First, the pattern search begins at the initial point $X_{0}$ that is given as a starting point by the user. At the first iteration, with a scalar $=1$ called mesh size, the pattern vectors are constructed as [0 1], [1 0], [-10], and [0-1], they may be called direction vectors. Then the pattern search algorithm adds the direction vectors to the initial point $X_{0}$ to compute the following mesh points:

$X_{0}+\left[\begin{array}{ll}1 & 0\end{array}\right]$

$X_{0}+\left[\begin{array}{ll}0 & 1\end{array}\right]$

$X_{0}+\left[\begin{array}{ll}-1 & 0\end{array}\right]$

$X_{0}+\left[\begin{array}{ll}0 & -1\end{array}\right]$

Fig. 2 illustrates the formation of the mesh and pattern vectors. The algorithm computes the objective function at the mesh points in the order shown.

The algorithm polls the mesh points by computing their objective function values until it finds one whose value is smaller than the objective function value of $X_{0}$. If there is such point, then the poll is successful and the algorithm sets this point equal to $X_{1}$.

After a successful poll, the algorithm steps to iteration 2 and multiplies the current mesh size by 2 , (this is called the expansion factor and has a default value of 2). The mesh at iteration 2 contains the following points: $2^{*}[10]+X_{1}$, $2^{*}[01]+X_{1}, 2^{*}[-10]+X_{1}$ and $2^{*}[0-1]+X_{1}$. The algorithm polls the mesh points until it finds one whose value is smal-

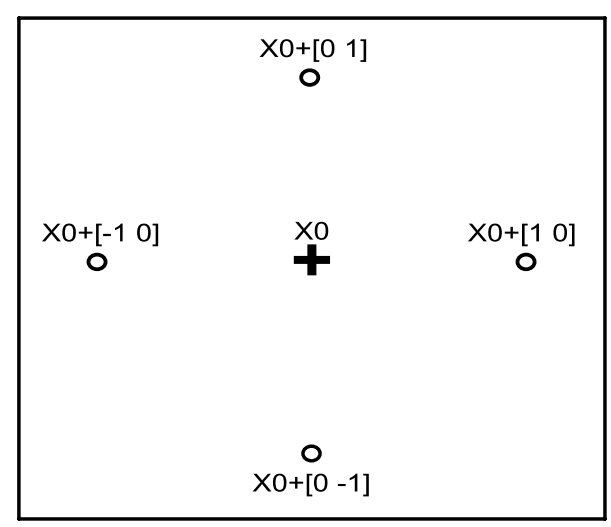

Fig. 2. PS mesh points and the pattern. ler than the objective function value of $X_{1}$. The first such point it finds is called $X_{2}$, and the poll is successful. Because the poll is successful, the algorithm multiplies the current mesh size by 2 to get a mesh size of 4 at the third iteration because the expansion factor $=2$.

Secondly, if iteration 3, (mesh size $=4$ ), ends up being unsuccessful poll, i.e. none of the mesh points has a smaller objective function value than the value at $X_{2}$, so the poll is called an unsuccessful poll. In this case, the algorithm does not change the current point at the next iteration. That is, $X_{3}=X_{2}$. At the next iteration, the algorithm multiplies the current mesh size by 0.5 , a contraction factor, so that the mesh size at the next iteration is smaller. The algorithm then polls with a smaller mesh size.

The pattern search optimization algorithm will repeat the illustrated steps until it finds the optimal solution for the minimization of the objective function. The algorithm stops when any of the following conditions occurs:

- The mesh size is less than mesh tolerance.

- The number of iterations performed by the algorithm reaches a predefined value.

- The total number of objective function evaluations performed by the algorithm reaches a pre-set maximum number of function evaluations.

- The distance between the point found at one successful poll and the point found at the next successful poll is less than a set tolerance.

- The change in the objective function from one successful poll to the next successful poll is less than a function tolerance.

The above steps and how PS evolves are depicted by the flow chart of Fig. 3. It should be noted that all the parameters involved in the pattern search optimization algorithm can be pre-defined subject to the nature of the problem being solved.

The PS operator gives the user a great deal of control regarding the direction of the search. After conducting a large number of experiments for many values of expansion and contraction factor, the best combination was found to be 2 and 0.5 respectively, giving an average best performance of optimal speed of computation as well as the right direction of the search.

\subsection{Constraint handling}

Many ideas have been suggested to insure that the solution will satisfies the constraint [15]. For example, the constraint can be augmented with the objective function using Lagrange multipliers. In this way the size of the problem will increase by introducing new parameters. In this study, the pattern search (PS) method handles constraints by using augmented Lagrangian to solve the non-linear constrained economic dispatch problem [16-19]. The variables' bounds and linear constraints are handled separately from non-linear constraints. Thus a sub-problem is formulated 


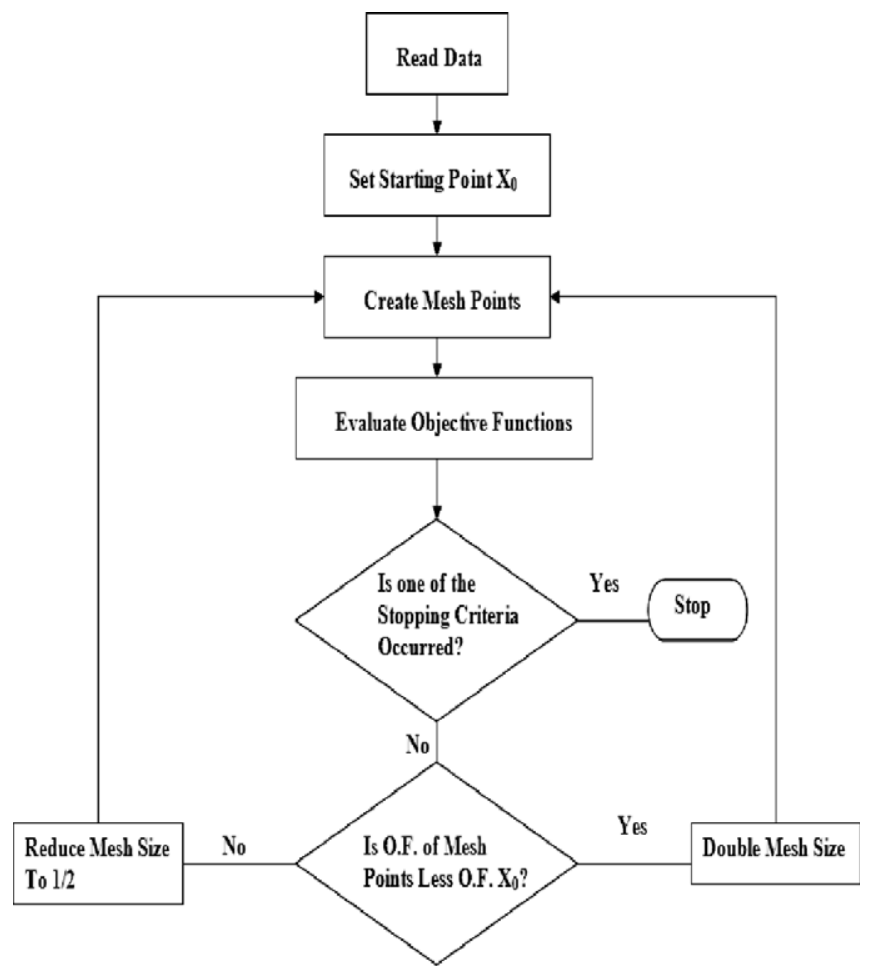

Fig. 3. Flow chart of pattern search.

and solved, (having the objective function and non-linear constraint function), using the Lagrangian and the penalty factors. Such sub-problem is minimized using a pattern search method, where that the linear constraints and bounds are satisfied. For more explanation on how PS handles constraints refer to $[18,20,21]$.

\section{Numerical results}

In order to asses the effectiveness and robustness of the proposed pattern search method, several test cases of economic load dispatch with valve-point effect have been considered. For simplicity, transmission losses are ignored in all test cases ( $P_{\mathrm{L}}$ in Eq. (3) is set to zero). The non-linear minimization problem formulation of all test cases has been solved using the predefined function pattern search incorporated in the GA\&DS toolbox of Matlab [21]. This function implements the pattern search algorithm described in Section 3. Thus, cost coefficients of the fuel cost and the combined objective function for the considered test cases were coded in Matlab environment.

Initially, several runs have been carried out with different values of the key parameters of PS such as the initial mesh size and the mesh expansion and contraction factors. In this study, the mesh size and the mesh expansion and contraction factor are selected as 1,2 and 0.5, respectively. In addition, a vector of initial points, i.e. $X_{0}$, was randomly generated (each initial point is bounded within the generators limits) to provide an initial guess for the PS to proceed. As for the stopping criteria, all tolerances were set to $10^{-6}$
Table 1

Generator loading and fuel cost determined by PS with total load demand of $850 \mathrm{MW}$

\begin{tabular}{ll}
\hline Generator & Generator production (MW) \\
\hline $\mathrm{Pg}_{1}$ & 300.2663 \\
$\mathrm{Pg}_{2}$ & 149.7331 \\
$\mathrm{Pg}_{3}$ & 399.9996 \\
$\Sigma \mathrm{Pg}_{i}=850 \mathrm{MW}$ & Total cost: $\$ 8234.05$ \\
\hline
\end{tabular}

Table 2

Comparison of PS, GA and EP

\begin{tabular}{llllll}
\hline $\begin{array}{l}\text { Evolution } \\
\text { method }\end{array}$ & $\begin{array}{l}\text { Mean } \\
\text { time }(\mathrm{s})\end{array}$ & $\begin{array}{l}\text { Best } \\
\text { time }(\mathrm{s})\end{array}$ & $\begin{array}{l}\text { Mean } \\
\text { cost }(\$)\end{array}$ & $\begin{array}{l}\text { Maximum } \\
\text { cost }(\$)\end{array}$ & $\begin{array}{l}\text { Minimum } \\
\text { cost }(\$)\end{array}$ \\
\hline GAB & 35.80 & 32.46 & - & - & 8234.08 \\
GAF & 24.65 & 23.03 & - & - & 8234.07 \\
CEP & 20.46 & 18.35 & 8235.97 & 8241.83 & 8234.07 \\
FEP & 4.45 & 3.79 & 8234.24 & 8241.78 & 8234.07 \\
MFEB & 8.00 & 6.31 & 8234.71 & 8241.80 & 8234.08 \\
IFEP & 6.78 & 6.11 & 8234.16 & 8234.54 & 8234.07 \\
PS & 0.81 & 0.62 & 8352.41 & 8453.00 & 8234.05 \\
\hline
\end{tabular}

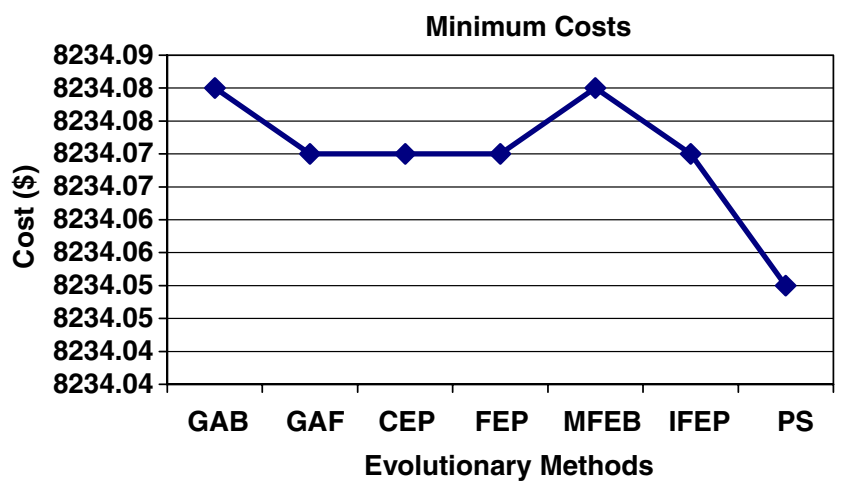

Fig. 4. Minimum cost comparison.

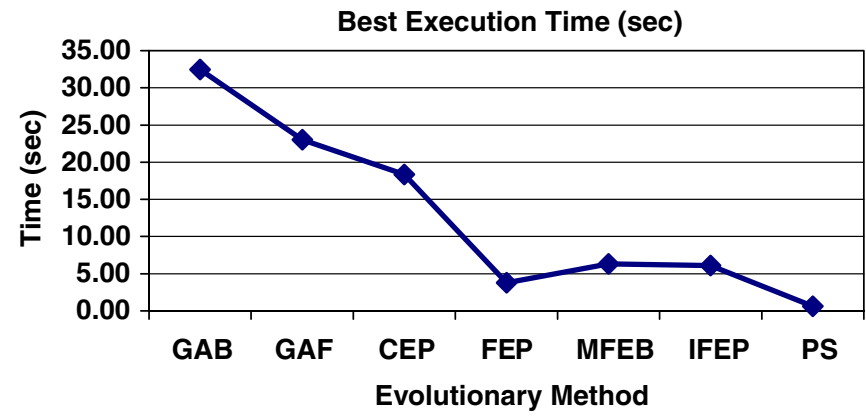

Fig. 5. Best execution time comparison.

and the maximum number of iterations and function evaluations were set to 1000 . All runs have been conducted on a modest $1 \mathrm{GHz}$ Pentium 3 processor with $256 \mathrm{MB}$ of 


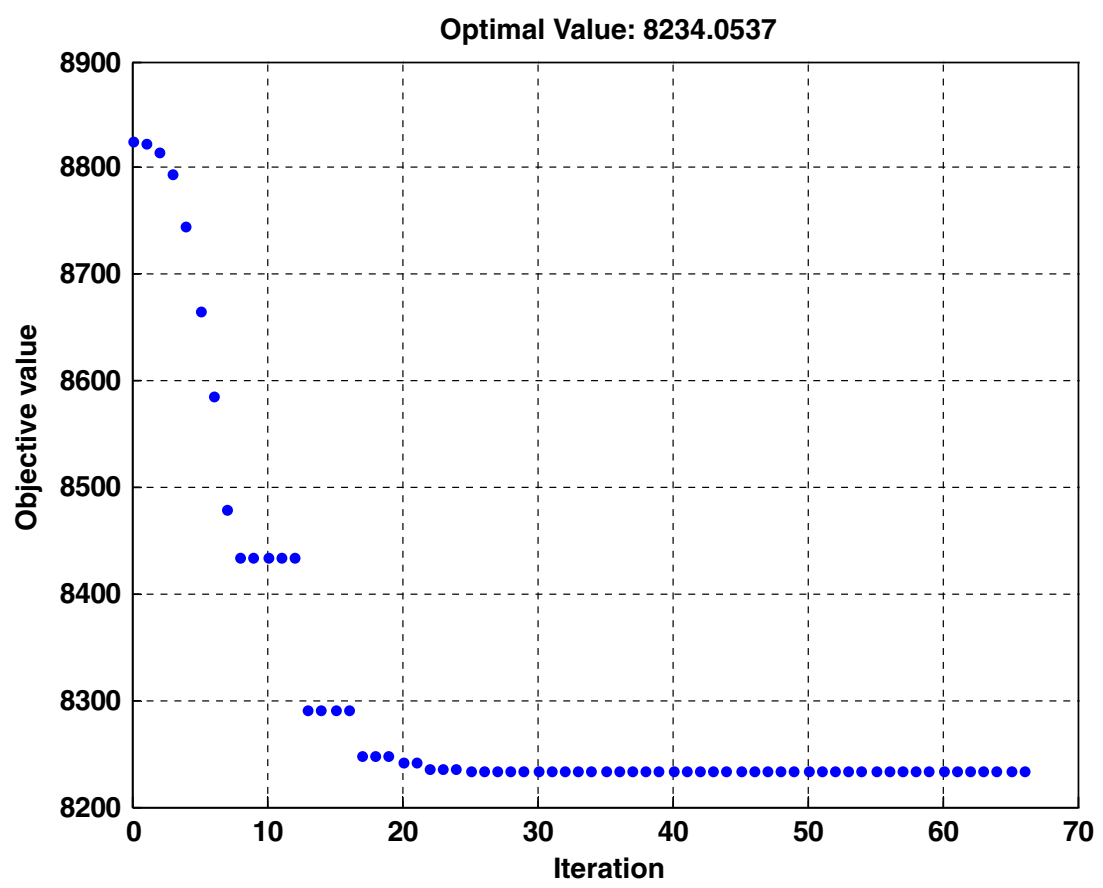

Fig. 6. Convergence of PS for the three-generating units.

RAM laptop computer, so the comparisons of computing times with those given in literature should be fair.

\subsection{Case I: three-generating units}

This test case consists of three generating units with quadratic cost function combined with the effects of valvepoint loading. The units data (upper and lower bounds) along with the cost coefficients for the fuel cost $(a, b, c, e$, and $f$ ) for the three generators with valve-point loading are given in $[6,22]$.

The pattern search algorithm has been executed for 100 with different starting points to study its performance and effectiveness. The solution of PS method and the execution time for a 100 runs were compared with the outcome of other evolutionary methods, for example Genetic

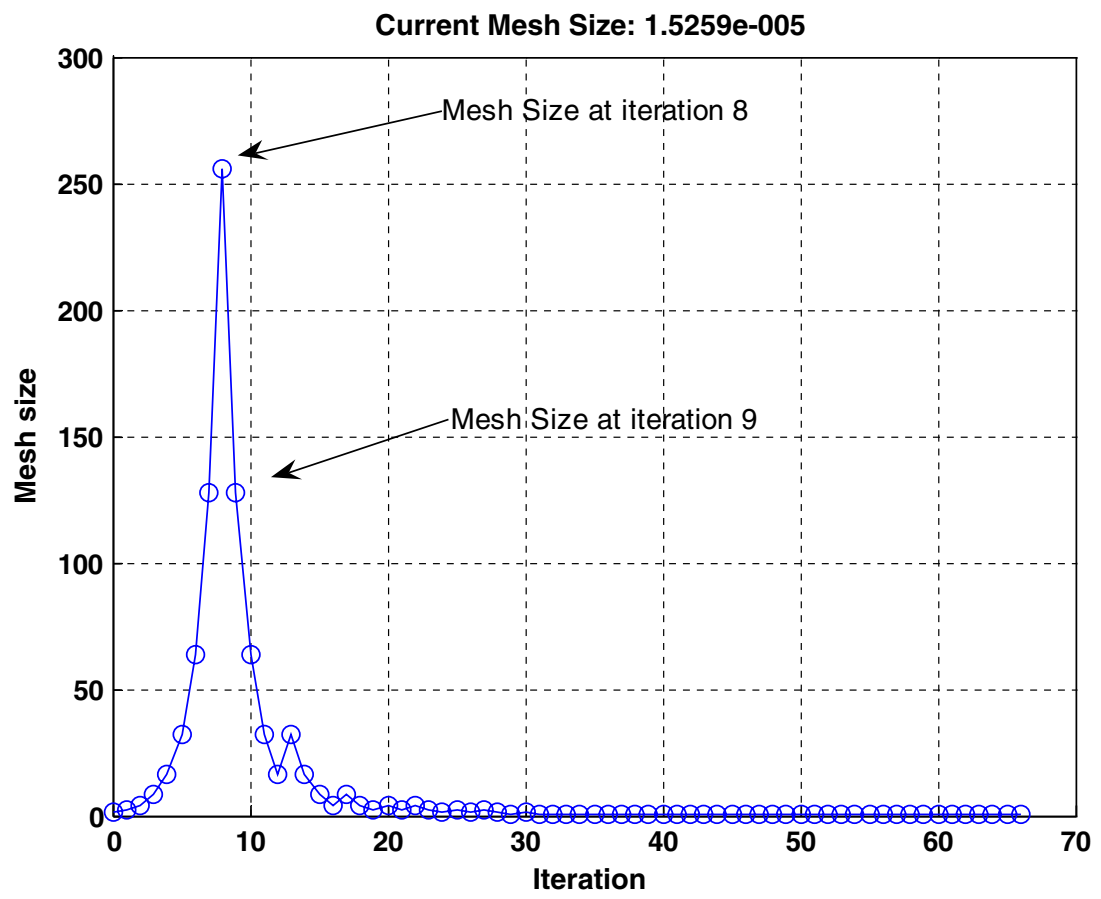

Fig. 7. Convergence of PS mesh size for the three-generating units. 


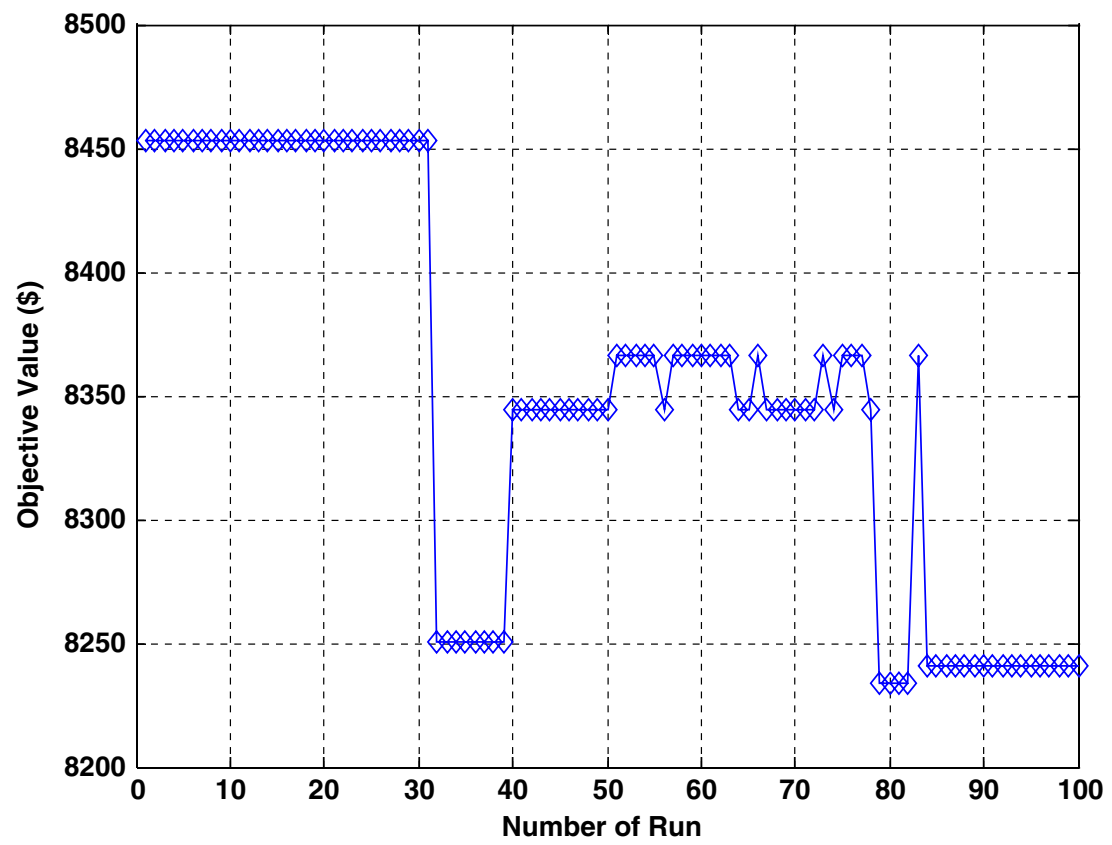

Fig. 8. Objective function value for 100 different starting point (case I).

Table 3

Generator loading and fuel cost determined by PS with total load demand of $1800 \mathrm{MW}$

\begin{tabular}{ll}
\hline Generator & Generator production $(\mathrm{MW})$ \\
\hline $\mathrm{Pg}_{1}$ & 538.5587 \\
$\mathrm{Pg}_{2}$ & 224.6416 \\
$\mathrm{Pg}_{3}$ & 149.8468 \\
$\mathrm{Pg}_{4}$ & 109.8666 \\
$\mathrm{Pg}_{5}$ & 109.8666 \\
$\mathrm{Pg}_{6}$ & 109.8666 \\
$\mathrm{Pg}_{7}$ & 109.8666 \\
$\mathrm{Pg}_{8}$ & 109.8666 \\
$\mathrm{Pg}_{9}$ & 109.8666 \\
$\mathrm{Pg}_{10}$ & 77.4666 \\
$\mathrm{Pg}_{11}$ & 40.2166 \\
$\mathrm{Pg}_{12}$ & 55.0347 \\
$\mathrm{Pg}_{13}$ & 55.0347 \\
$\Sigma \mathrm{Pg}_{i}=1800 \mathrm{MW}$ & Total cost: $\$ 17969.17$ \\
\hline
\end{tabular}

Table 4

Comparison of PS, GA and EP

\begin{tabular}{llllll}
\hline $\begin{array}{l}\text { Evolution } \\
\text { method }\end{array}$ & $\begin{array}{l}\text { Mean } \\
\text { time }(\mathrm{s})\end{array}$ & $\begin{array}{l}\text { Best } \\
\text { time }(\mathrm{s})\end{array}$ & $\begin{array}{l}\text { Mean } \\
\text { cost }(\$)\end{array}$ & $\begin{array}{l}\text { Maximum } \\
\text { cost }(\$)\end{array}$ & $\begin{array}{l}\text { Minimum } \\
\text { cost }(\$)\end{array}$ \\
\hline CEP & 294.96 & 293.41 & 18190.32 & 18404.04 & 18048.21 \\
FEP & 168.11 & 166.43 & 18200.79 & 18453.82 & 18018.00 \\
MFEP & 317.12 & 315.98 & 18192.00 & 18416.89 & 18028.09 \\
IFEP & 157.43 & 156.81 & 18127.06 & 18267.42 & 17994.07 \\
PS & 5.88 & 1.65 & 18088.84 & 18233.52 & 17969.17 \\
\hline
\end{tabular}

Algorithm (GA) and Evolutionary Programming (EP), applied to the same test system in [22]. This experimentation compares the performance of PS with the other methods in terms of dispatching cost and convergence speed. Table 1 shows the optimal solutions determined by PS

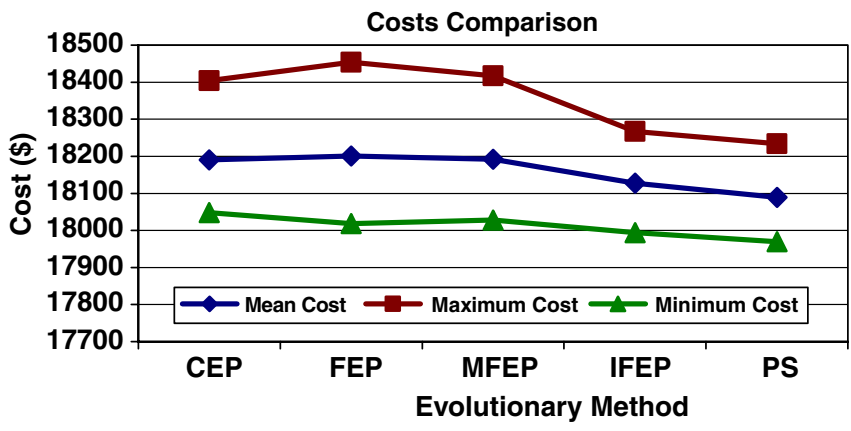

Fig. 9. Minimum cost comparison case II.

for the three units, while the execution time and cost comparison are shown in Table 2. The definition of the various methods (GAB, GAF, etc) may be found in [22].

All methods give a similar 'best' solution, whereas 'mean' and 'maximum' costs differ. The PS algorithm is significantly faster than methods described in [22]. Figs. 4 and 5 compare the results of the methods in

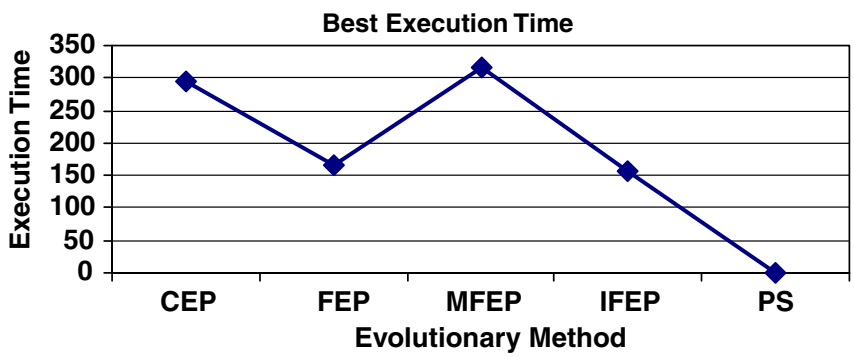

Fig. 10. Best execution time comparison case II. 


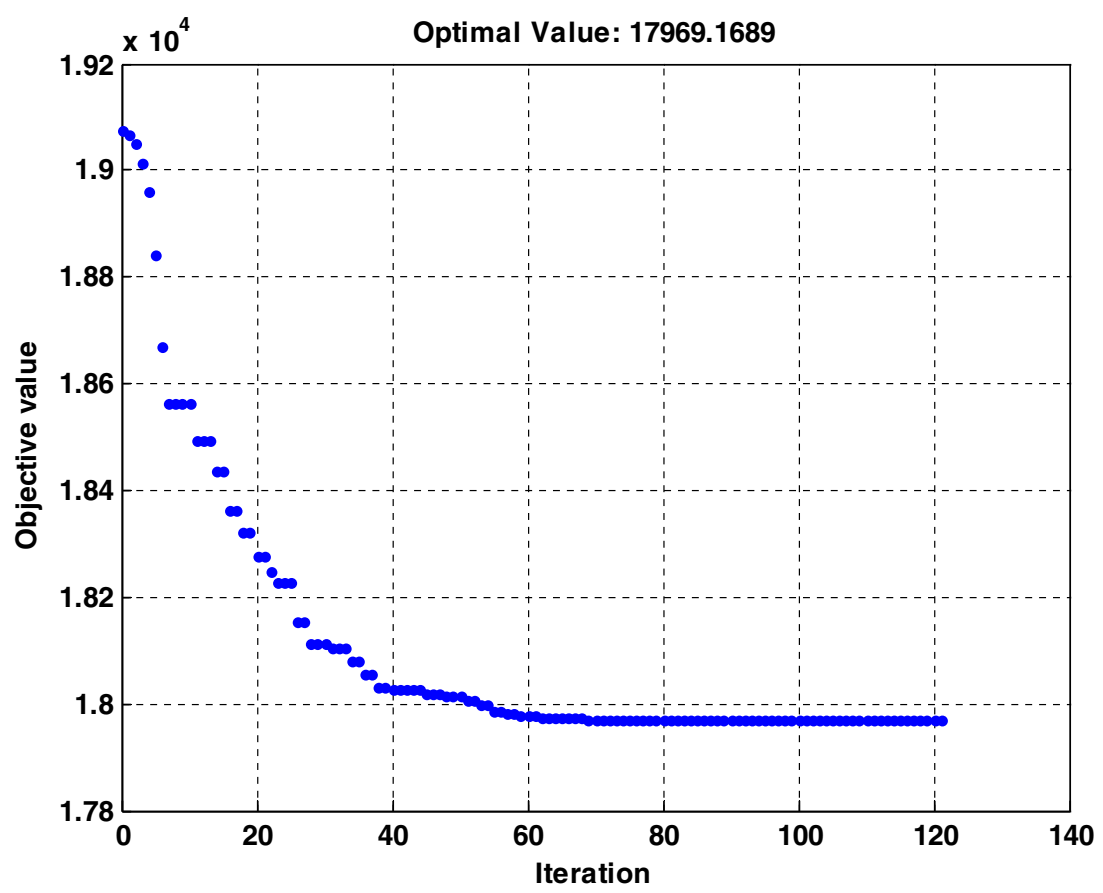

Fig. 11. Convergence of PS for the 13-generating units.

terms of minimum cost and best execution time, respectively.

The convergence of optimal solution using PS is shown in Fig. 6, where only about 22 iterations were needed to find the optimal solution. However, PS may be allowed to continue the search in the neighborhood of the optimal point to increase the confidence in the result. PS stops after 44 more iteration and returns the optimal value.
Fig. 7 depicts the mesh size through out the convergence process. It is apparent form the figure that the mesh size decreases until the algorithm terminates, in this case at mesh size $1.5259 \mathrm{e}-005$ which is more that the giving as stopping criteria, thus indicating that this particular run did not terminate using the mesh size tolerance. Fig. 7 shows that for the first 8 iteration the poll was successful since the mesh size keeps increasing as the algorithm had

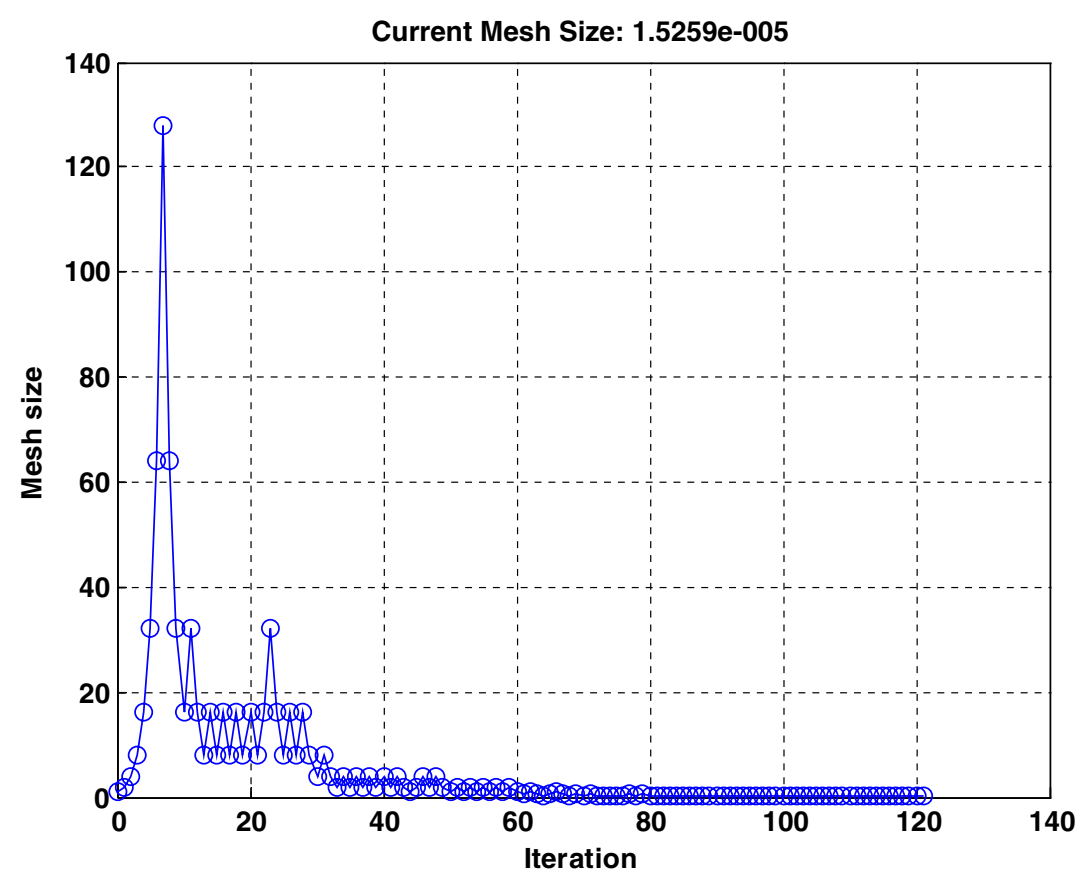

Fig. 12. Convergence of PS mesh size for the 13-generating units. 


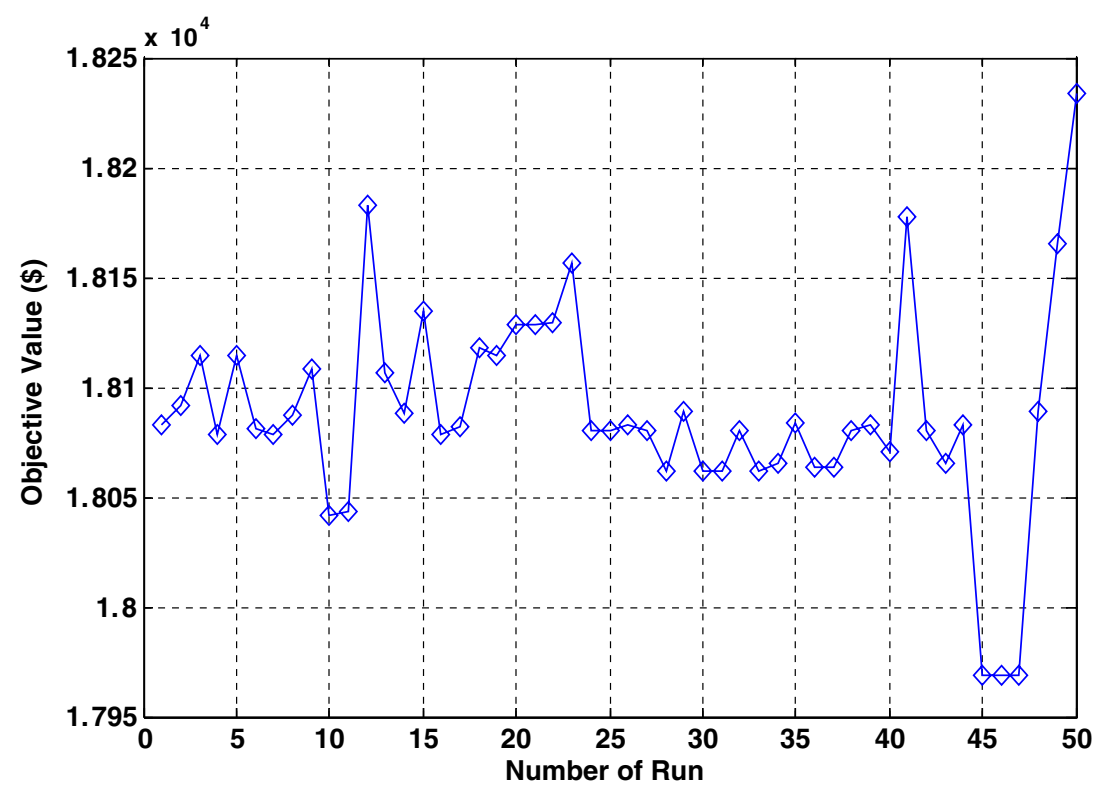

Fig. 13. Objective function value for 50 different starting point (case II).

Table 5

Generator loading and fuel cost determined by PS with total load demand of 10,500 MW

\begin{tabular}{|c|c|c|c|c|c|c|c|}
\hline Generator & $\begin{array}{l}\text { Generator production } \\
\text { (MW) }\end{array}$ & Generator & $\begin{array}{l}\text { Generator production } \\
\text { (MW) }\end{array}$ & Generator & $\begin{array}{l}\text { Generator production } \\
\text { (MW) }\end{array}$ & Generator & $\begin{array}{l}\text { Generator production } \\
\text { (MW) }\end{array}$ \\
\hline $\mathrm{Pg}_{1}$ & 110.8051 & $\mathrm{Pg}_{11}$ & 168.8008 & $\mathrm{Pg}_{21}$ & 523.2793 & $\mathrm{Pg}_{31}$ & 189.9989 \\
\hline $\mathrm{Pg}_{2}$ & 110.8051 & $\mathrm{Pg}_{12}$ & 168.8008 & $\mathrm{Pg}_{22}$ & 523.2793 & $\mathrm{Pg}_{32}$ & 189.9989 \\
\hline $\mathrm{Pg}_{3}$ & 97.40230 & $\mathrm{Pg}_{13}$ & 214.7606 & $\mathrm{Pg}_{23}$ & 523.2832 & $\mathrm{Pg}_{33}$ & 189.9989 \\
\hline $\mathrm{Pg}_{4}$ & 179.7332 & $\mathrm{Pg}_{14}$ & 304.5204 & $\mathrm{Pg}_{24}$ & 523.2832 & $\mathrm{Pg}_{34}$ & 164.8036 \\
\hline $\mathrm{Pg}_{5}$ & 92.70700 & $\mathrm{Pg}_{15}$ & 394.2801 & $\mathrm{Pg}_{25}$ & 523.2793 & $\mathrm{Pg}_{35}$ & 164.8036 \\
\hline $\mathrm{Pg}_{6}$ & 140.0000 & $\mathrm{Pg}_{16}$ & 394.2801 & $\mathrm{Pg}_{26}$ & 523.2793 & $\mathrm{Pg}_{36}$ & 164.8036 \\
\hline $\mathrm{Pg}_{7}$ & 259.6004 & $\mathrm{Pg}_{17}$ & 489.2801 & $\mathrm{Pg}_{27}$ & 10.00080 & $\mathrm{Pg}_{37}$ & 109.9989 \\
\hline $\mathrm{Pg}_{8}$ & 284.6004 & $\mathrm{Pg}_{18}$ & 489.2801 & $\mathrm{Pg}_{28}$ & 10.00280 & $\mathrm{Pg}_{38}$ & 109.9989 \\
\hline $\mathrm{Pg}_{9}$ & 284.6004 & $\mathrm{Pg}_{19}$ & 511.2817 & $\mathrm{Pg}_{29}$ & 10.00280 & $\mathrm{Pg}_{39}$ & 109.9989 \\
\hline $\operatorname{Pg}_{10}$ & 130.0028 & $\mathrm{Pg}_{20}$ & 511.2817 & $\mathrm{Pg}_{30}$ & 87.80080 & $\mathrm{Pg}_{40}$ & 511.2817 \\
\hline \multicolumn{4}{|c|}{$\Sigma \mathrm{Pg}_{i}=10,500 \mathrm{MW}$} & \multicolumn{4}{|c|}{ Total cost: $\$ 121415.14$} \\
\hline
\end{tabular}

to expand the scope of the search. This is accomplished by multiplying the current mesh size by the expansion factor, in this study taken as 2 . This scenario continued until iteration number 8 when the mesh size reached 256. At iteration number 9 the mesh size decreased by half due to multiplying the current mesh size by the contracting factor, indicating an unsuccessful poll in the previous iteration. This process continues until reaching one of the termination criteria.

It is worth mentioning that the mean and the maximum costs are higher than those of the other methods, and this is a certain drawback of the performance of PS in this test. Moreover, it has been observed that the algorithm is quite sensitive to the initial (starting) point and how far it is from the global optimal solution. Fig. 8 illustrates the sensitivity of PS where a hundred solutions were obtained by PS with different initial values. The optimal solution has been reached a number of times for initial points around run number 80 . The total execution time for the 100 runs was $80.75 \mathrm{~s}$. Other quality answers occurred for runs between
32-40 and 84-100. However, there were also several less successful results as illustrated in Fig. 8.

\subsection{Case II: 13-generating units}

This test case consists of 13-generating units with quadratic cost function combined with the effects of valvepoint loading. The units data (upper and lower bounds) along with the cost coefficients for the fuel cost $(a, b, c, e$,

Table 6

Comparison of PS, GA and EP

\begin{tabular}{llllll}
\hline $\begin{array}{l}\text { Evolution } \\
\text { method }\end{array}$ & $\begin{array}{l}\text { Mean } \\
\text { time }(\mathrm{s})\end{array}$ & $\begin{array}{l}\text { Best } \\
\text { time }(\mathrm{s})\end{array}$ & $\begin{array}{l}\text { Mean } \\
\text { cost }(\$)\end{array}$ & $\begin{array}{l}\text { Maximum } \\
\text { cost }(\$)\end{array}$ & $\begin{array}{l}\text { Minimum } \\
\text { cost }(\$)\end{array}$ \\
\hline CEP & 1956.93 & 1955.20 & 124793.48 & 126902.89 & 123488.29 \\
FEP & 1039.16 & 1037.90 & 124119.37 & 127245.59 & 122679.71 \\
MFEP & 2196.10 & 2194.70 & 123489.74 & 124356.47 & 122647.57 \\
IFEP & 1167.35 & 1165.70 & 123382.00 & 125740.63 & 122624.35 \\
PS & 42.98 & 12.66 & 122332.65 & 125486.29 & 121415.14 \\
\hline
\end{tabular}




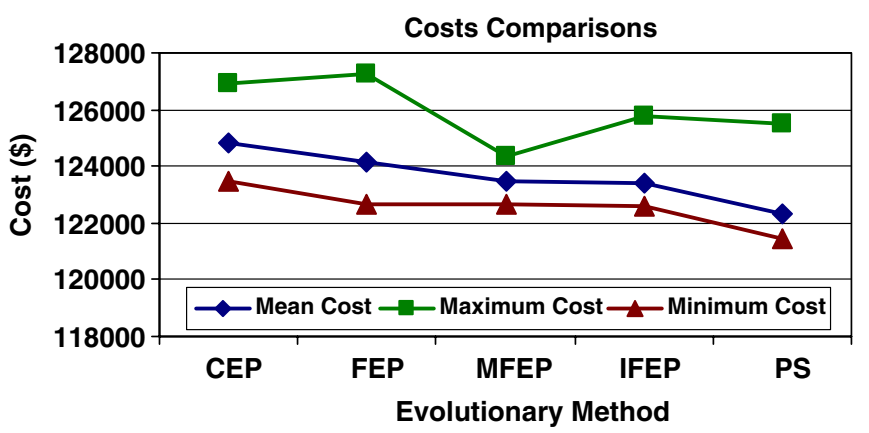

Fig. 14. Costs comparison case III.

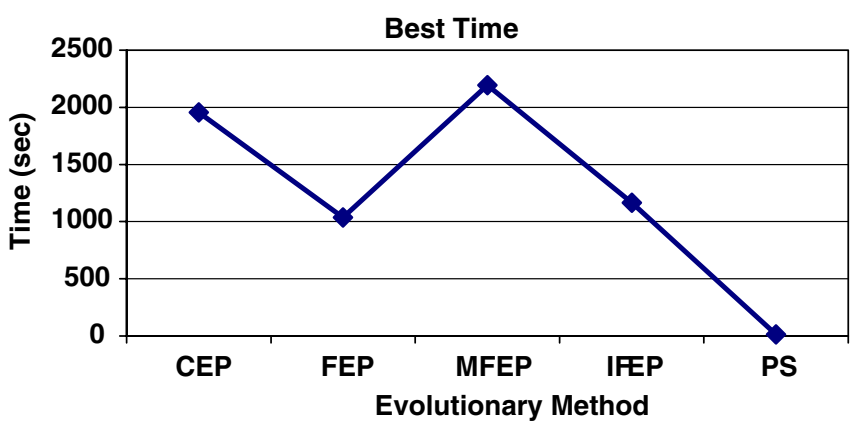

Fig. 15. Best time comparison case III.

and $f$ ) for the 13 generators with valve-point loading are given in $[22,23]$.

The pattern search algorithm has been executed 50 times with different starting points and similar comparisons as for Case I are summarized by Tables 3 and 4 . The results for all the 'EP' methods are taken from [22,21].

In this case the PS method outperforms all other algorithms in terms of all costs: minimum, mean and maximum, while at the same time offering significant saving in computing times (see Figs. 9 and 10).

The convergence of the PS algorithm is shown in Fig. 11. As before, the search continues beyond the 70 iterations (when the optimal solution has been reached) to improve the confidence in the result. A total of 122 iterations have been performed.

The dynamics of the mesh size is depicted by Fig. 12. As before, the initial polling is successful leading to mesh size increases, whereas subsequently the mesh size is being reduced (with the exception of iterations 11 and 23) indicating unsuccessful polls. As in Case I, the termination criteria for the mesh size has not been reached.

Although the PS has achieved the 'best' optimum only on three occasions out of 50 runs (see Fig. 13), the overall minimum and mean costs are still better than those obtained by other methods. The total execution time for 50 runs is $294.06 \mathrm{~s}$, which is comparable to just one run using the other techniques.

\subsection{Case III: 40-generating units}

This test case consists of 40 generating units with quadratic cost function combined with the effects of valvepoint loading, with full data given in $[22,24]$. The pattern search algorithm has been executed for a hundred times with different starting points and results and comparisons

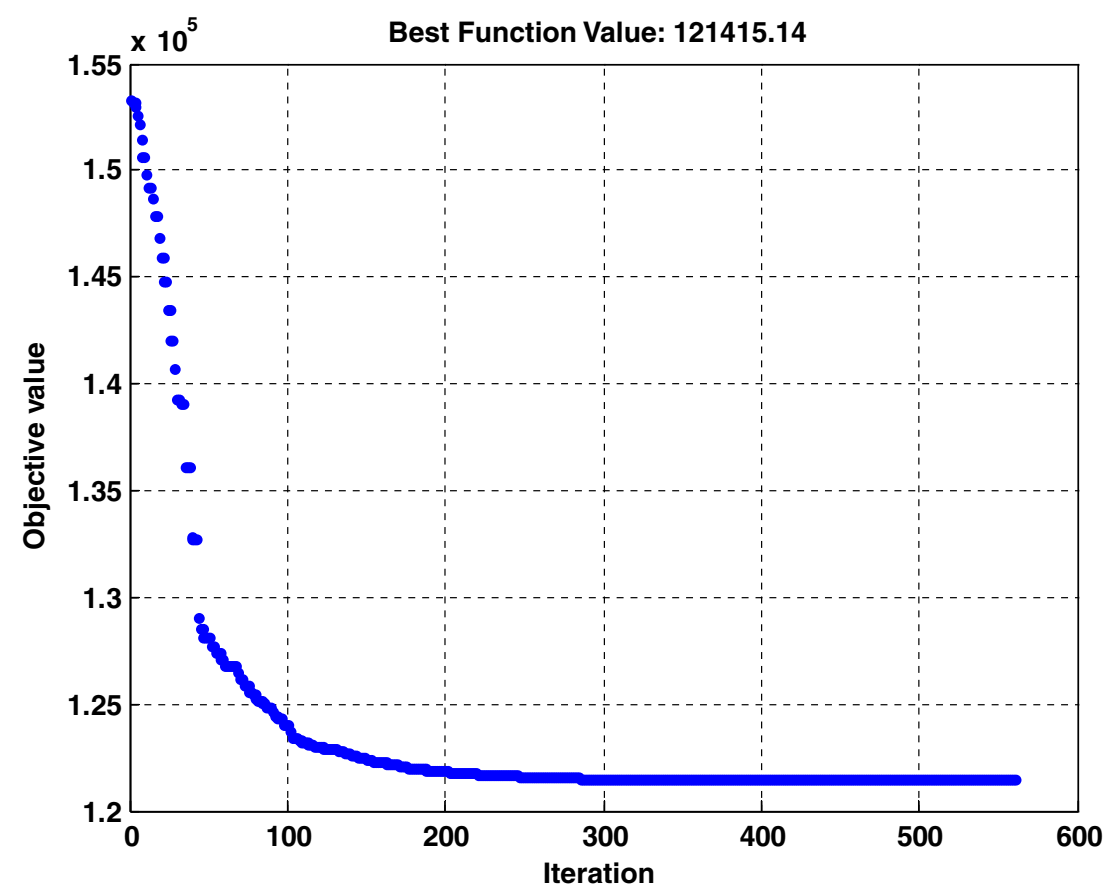

Fig. 16. Convergence of PS for the 40-generating units. 


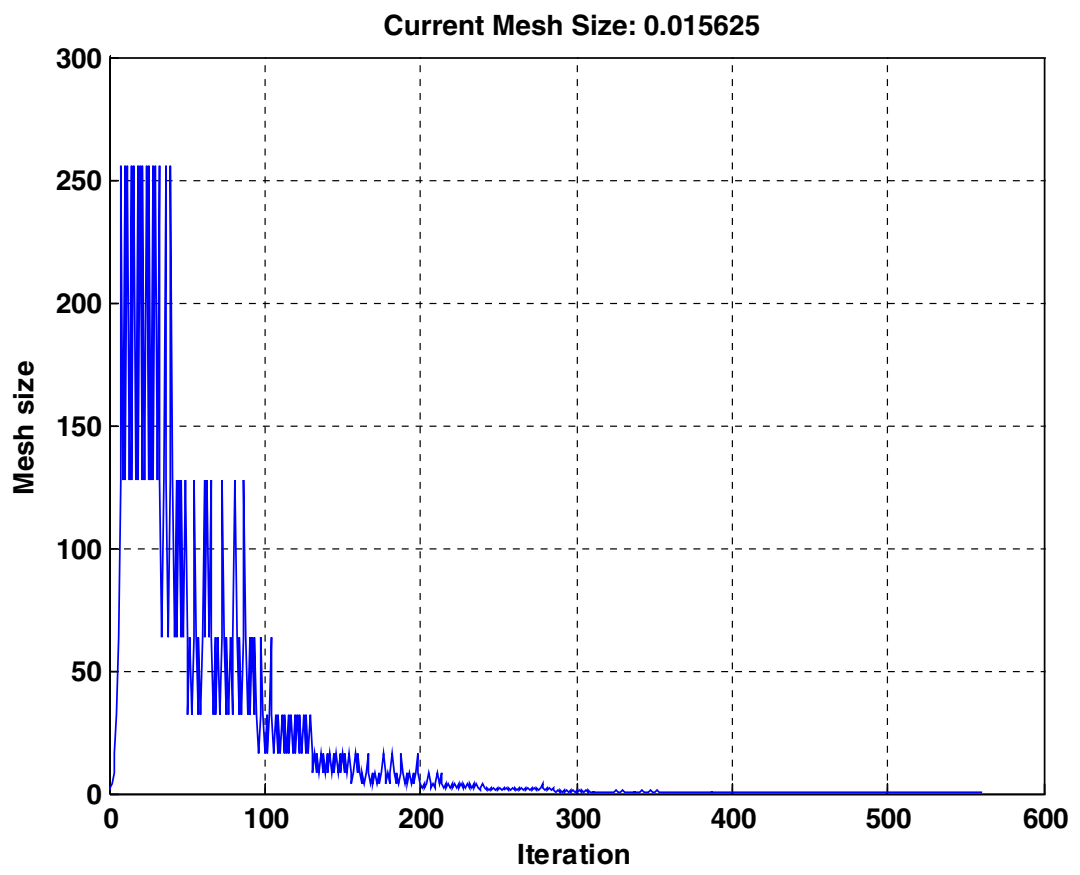

Fig. 17. Convergence of PS mesh size for the 40-generating units.

with other methods are given in Tables 5 and 6 , respectively.

Figs. 14 and 15 shows the comparison of costs and best time for all methods.

Figs. 16-18 show the convergence of the objective function, changes to mesh size and quality of the optimum depending on the starting point. The tendencies and the properties of the algorithm are similar to those observed when studying Case II. Overall, the PS method provides the best minimum and mean costs of all the methods compared at significant savings of computational effort. These short computing times allow for more cases to be studied with the aim of increasing the confidence in the final solution.

The way that PS works is that after each successful pole (finding a lower objective function value for the mesh

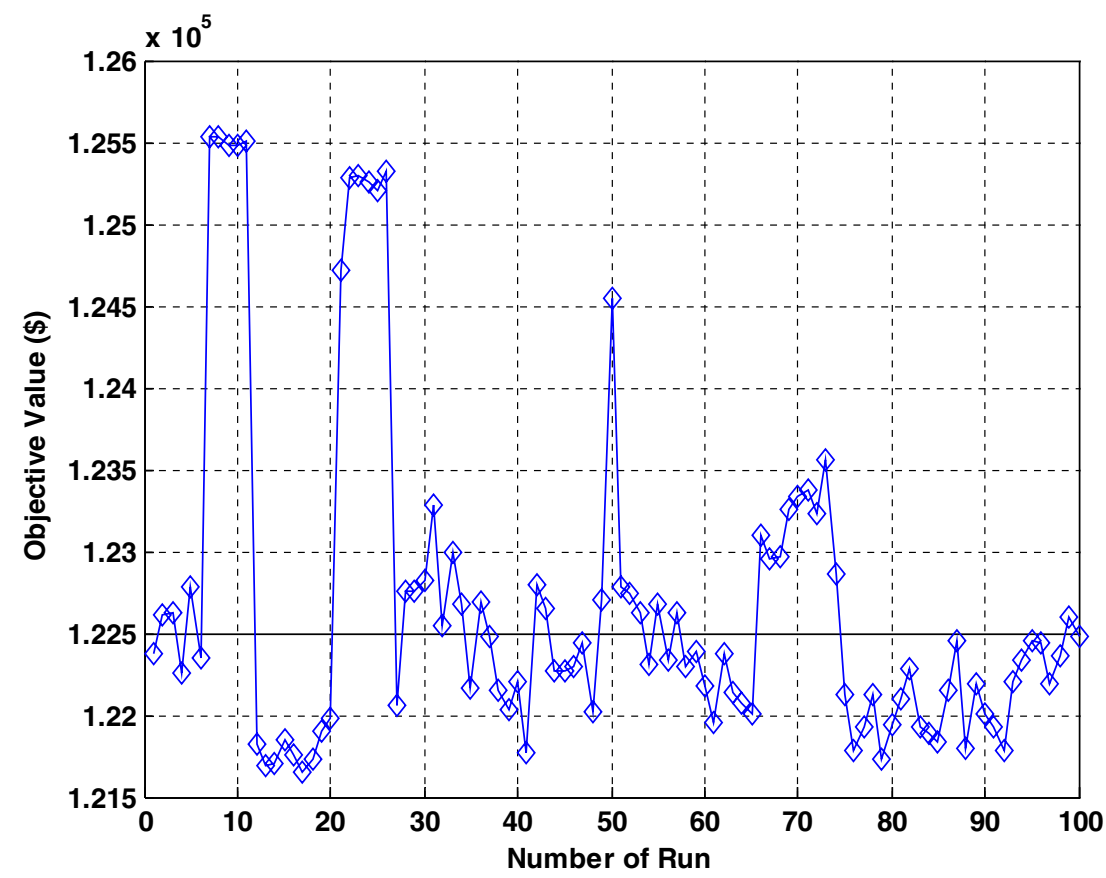

Fig. 18. Objective function values for 100 different starting points. 
points) the algorithm expands the mesh size to assure that there are no lower objective functions for other points in the region. Referring to the figures that show the mesh sizes it can be observed that the PS may reach a very large mesh size around the current point just to prevent the method from becoming stuck in a local minimum or a saddle point. For example, in Fig. 17, PS goes to 256 in many iterations rejecting all local minimums and saddle points.

\section{Conclusions}

This paper introduces a new approach based on pattern search (PS) optimization to study the power system economic dispatch with valve-point effect, which is formulated as a constrained optimization problem. The proposed method has been applied to three test cases. When compared with evolutionary programming (EP), and in one case also with a genetic algorithm (GA), the analysis results have demonstrated that PS outperforms the other methods in terms of reaching a better optimal solution and significant reduction of computing times. On the other hand, the PS is more sensitive to the initial guess and appears to rely on how close the given initial point is to the global solution. This makes the PS method possibly more susceptible to getting trapped in local minima. However, the much improved speed of computation allows for additional searches to be made to increase the confidence in the solution. It should also be noted that GA and EP methods normally start with a population of starting points, rather than a single initial point like the PS, thus require even more computational effort. Overall, the PS algorithm has been shown to be very helpful in studying optimization problems in power systems.

\section{Acknowledgement}

This work has been supported by the government of The State of Kuwait, Public Authority of Applied Science and Training, Kuwait. Contract \# TS-06-02.

\section{References}

[1] Alder RB. Security consideration economic dispatch with participation factors. IEEE Transa Power App Syst 1977;PAS-96.

[2] Bui RT, Ghaderpanah S. Real power rescheduling and security assessment. IEEE Trans Power App Syst 1982;PAS-101:2906-15.

[3] Pancholi RK, Swarup KS. Particle swarm optimization for security constrained economic dispatch. In: Presented at International Con- ference on Intelligent Sensing and Information Processing. (IEEE Cat. No. 04EX783), Chennai, India; 2004.

[4] EL-Sharkawy M, Nieebur D. Artificial neural networks with application to power systems. IEEE Power Eng Soc, A Tutorial Course 1996.

[5] Yalcinoz T, Short MJ. Neural networks approach for solving economic dispatch problem with transmission capacity constraints. IEEE Trans Power Syst 1998;13:307-13.

[6] Walters DC, Sheble GB. Genetic algorithm solution of economic dispatch with valve-point loading. Power Syst, IEEE Trans 1993;8:1325-32.

[7] Youssef HK, El-Naggar KM. Genetic based algorithm for security constrained power system economic dispatch. Elect Power Syst Res 2000;53:47-51.

[8] Chen CL, Chen N. Direct search method for solving economic dispatch problem considering transmission capacity constraints. IEEE Trans Power Syst 2001;16:764-9.

[9] Coelho LS, Mariani VC. Combining of chaotic differential evolution and quadratic programming for economic dispatch optimization with valve-point effect. Power Syst, IEEE Trans 2006;21: 989-96.

[10] Hooke R, Jeeves TA. Direct search solution of numerical and statistical problems. J Associat Comput Machin 1961;8:212-29.

[11] Lewis RM, Torczon V, Trosset MW. Direct search methods: then and now. J Comput Appl Math 2000;124:191-207.

[12] Kim JO, Shin D-J, Park J-N, Singh C. Atavistic genetic algorithm for economic dispatch with valve-point effect. Elect Power Syst Res 2002;62:201-7.

[13] Goldberg DE. Genetic algorithms in search, optimization, and machine learning. Reading, Mass, Harlow: Addison-Wesley; 1989.

[14] Michalewicz Z. Genetic algorithms + data structures = evolution programs. 3rd ed. Berlin, New York: Springer-Verlag; 1996.

[15] Youssef HK, El-Shibini M, Hazaa GA. Some new aspects in power system dynamic security using pattern recognition. presented at Second Middle East Power Conference. MEPCON-92, 1992.

[16] Lewis RM, Torczon V. Pattern search algorithms for linearly constrained minimization. SIAM J Optimiz 1999;10:917-41.

[17] Lewis RM, Torczon V. Pattern search algorithms for bound constrained minimization. SIAM J Optimiz 1999;9:1082-99.

[18] Lewis RM, Torczon V. A globally convergent augmented Lagrangian pattern search algorithm for optimization with general constraints and simple bounds. SIAM J Optimiz 2001;12:1075-89.

[19] Torczon V. On the convergence of pattern search algorithms. SIAM J Optimiz 1997;7:1-25.

[20] Conn AR, Gould NIM, Toint PL. A globally convergent augmented Lagrangian algorithm for optimization with general constraints and simple bounds. SIAM J Numer Anal 1991;28:545-72.

[21] Genetic Algorithm and Direct Search Toolbox for use with Matlab user's guide. 2 ed. The Math works inc.

[22] Sinha N, Chakrabarti R, Chattopadhyay PK. Evolutionary programming techniques for economic load dispatch. Evol Comput, IEEE Trans 2003;7:83-94.

[23] Wong KP, Wong YW. Thermal generator scheduling using hybrid genetic simulated-annealing. Gener, Transmiss Distrib, IEE Proceed 1995; 142:372-80.

[24] Chen P-H, Chang H-C. Large-scale economic dispatch by genetic algorithm. Power Syst, IEEE Trans 1995;10:1919-26. 